\title{
VARIETIES OF TOPOLOGICAL GROUPS AND LEFT ADJOINT FUNCTORS
}

\author{
Dedicated to the memory of Hanna Neumann
}

SIDNEY A. MORRIS

(Received 25 May 1972)

Communicated by J. B. Miller

\section{Introduction}

In [6] and [2] Markov and Graev introduced their respective concepts of a free topological group. Graev's concept is more general in the sense that every Markov free topological group is a Graev free topological group. In fact, if $F G(X)$ is the Graev free topological group on a topological space $X$, then it is the Markov free topological group $F M(Y)$ on some space $Y$ if and only if $X$ is disconnected. This, however, does not say how $F G(X)$ and $F M(X)$ are related.

We show that $F M(X)$ is isomorphic to the coproduct (in the category of all topological groups) of $F G(X)$ and the discrete group $Z$ of integers. This result is analagous to one announced by Ward [16] namely that the Markov free abelian topological group is isomorphic to the direct product of the Graev free abelian topological group and $Z$. Both results are special cases of the following:

If $\mathfrak{B}$ is a (non-indiscrete) variety of topological groups [7] and $F(X, \mathfrak{B})$ and $G(X, \mathfrak{B})$ are respectively the Markov and Graev free topological groups of $\mathfrak{B}$ on $X$, then $F(X, \mathfrak{B})$ is isomorphic to the coproduct in $\mathfrak{B}$ of $G(X, \mathfrak{B})$ and a onegenerator Markov free topological group of $\mathfrak{B}$.

As an immediate consequence of this we see that topological spaces with isomorphic Graev free topological groups have isomorphic Markov free topological groups. Another consequence is that every $G(X, \mathfrak{B})$ is projective in $\mathfrak{B}$. We also use the above result to answer a question of Nummela [14] on Markov free topological groups.

In [8] we introduced the concept of a $\beta$-variety as a variety for which the Markov free topological groups have some pleasant properties. We show that we obtain the same class of varieties if we based the definition of a $\beta$-variety on Graev free topological groups. This is noteworthy, since recent work [12] has 
shown (for example) that varieties generated by connected locally compact groups are $\beta$-varieties.

\section{Definitions and results}

Definition. A non-empty class $\mathfrak{B}$ of (not necessarily Hausdorff) topological groups is said to be a variety of topological groups if it is closed under the operations of taking subgroups, quotient groups, arbitrary cartesian products and isomorphic images.

Definition. Let $\mathfrak{B}$ be a variety, $X$ a topological space and $F(X, \mathfrak{B})$ a member of $\mathfrak{B}$. Then $F(X, \mathfrak{B})$ is said to be a Markov free topological group of $\mathfrak{B}$ on $X$ if it has the properties:

(a) there exists a mapping $\eta: X \rightarrow F(X, \mathfrak{B})$ such that $\eta: X \rightarrow \eta(X)$ is a homeomorphism,

(b) for any continuous mapping $\gamma$ of $X$ into any member $H$ of $\mathfrak{B}$, there exists a unique continuous homorphism $\Gamma$ of $F(X, V)$ into $H$ such that $\Gamma \eta=\gamma$.

Definition. Let $\mathfrak{B}$ be a variety, $X$ a topological space and $G(X, \mathfrak{B})$ a member of $\mathfrak{B}$. Distinguish in $X$ and arbitrary point $e$. Then $G(X, \mathfrak{B})$ is said to be a Graev free topological group of $\mathfrak{B}$ on $X$ if it has the properties:

(a) there exists a mapping $\eta: X \rightarrow G(X, \mathfrak{B})$ such that $\eta: X \rightarrow \eta(X)$ is a homeomorphism and $\eta(e)$ is the identity element of $G(X, \mathfrak{B})$,

(b) for any continuous mapping $\gamma$ of $X$ into any member $H$ of $\mathfrak{B}$ such that $\gamma(e)$ is the identity element of $\mathrm{H}$, there exists a unique continuous homomorphism $\Gamma$ of $G(X, \mathfrak{B})$ into $H$ such that $\Gamma \eta=\gamma$.

Definition. Let $\mathfrak{B}$ be a variety and $\left\{\mathrm{G}_{i}: i \in I\right\}$ a family of members of $\mathfrak{B}$. Then the topological group $F$ in $\mathfrak{B}$ is said to be a $\mathfrak{B}$-product of $\left\{G_{i}: i \in I\right\}$, denoted by $\bigsqcup_{2} G_{i}$, if it has the properties:

(a) for each $i \in I$, there exists a mapping $\eta_{i}: G_{i} \rightarrow F$ such that $\eta_{i}: G \rightarrow \eta_{i}(G)$ is an isomorphism,

(b) if for each $i \in I, \gamma_{i}$ is a continuous homomorphism of $G_{i}$ into any member $H$ of $\mathfrak{B}$ then there exists a unique continuous homorphism $\Gamma$ of $F$ into $H X$ such that $\Gamma \eta_{i}=\gamma_{i}$, for each $i$.

Every variety $\mathfrak{B}$ defines a complete category [1]: the objects are the members of $\mathfrak{B}$ and the morphisms are the continuous homomorphisms between members of $\mathfrak{B}$. ( $\mathfrak{B}$ has products and equalizers.) The forgetful functor $S: \mathfrak{B} \rightarrow$ Top (the category of topological spaces) preserves products and equalizers and is therefore continuous (preserves limits). The solution set condition is satisfied and Freyd's adjoint functor theorem shows that $S$ has a left adjoint $F:$ Top $\rightarrow \mathfrak{B}$. If $\eta: X \rightarrow S F X$ is the front adjunction, then for every continuous map $\gamma: X \rightarrow S H$, where $H \in \mathfrak{B}$, 
there exists a unique continuous homomorphism $\Gamma$ of $F X$ into $H$ such that $S(\Gamma) \eta=\gamma$. Further, it is clear that $\eta: X \rightarrow \eta(X)$ is a homeomorphism if and only if $V$ has a member with a subspace homeomorphic to $X$. Thus we have:

THEOREM 1. Let $\mathfrak{B}$ be a variety and $X$ a topological space. Then $F(X, \mathfrak{B})$ exists if and only if $\mathfrak{B}$ has a member with a subspace homeomorphic to $X$.

It is obvious that if $F(X, \mathfrak{B})$ exists, then it is unique, up to isomorphism. Noting that the class of groups of groups which, with some topology, appear in $\mathfrak{B}$ is a variety of groups [13] it is shown in [7] that $F(X, \mathfrak{B})$ is the free group on the set $\eta(X)$ of the underlying variety of groups. In particular, $\eta(X)$ generates $F(X, \mathfrak{B})$ algebraically. This distinguishes varietal categories from other categories of topological groups. For example, if $C$ is the category of compact groups, then the forgetful functor $S: C \rightarrow$ Top has a left adjoint $F:$ Top $\rightarrow C$. However for $X \in$ Top and $\eta$ the front adjunction: $X \rightarrow S F X$, it is not true that $\eta(X)$ generates $F X$ algebraically. Rather, the subgroup generated by $\eta(X)$ is dense in $F X$. (For further comments see [11].)

Let us denote by Top p $_{0}$ the category of pointed spaces; the objects are $\left(X, x_{0}\right)$ with $x_{0} \in X \in$ Top and the morphisms are base point preserving continuous maps. There is a forgetful functor $S_{0}: \mathfrak{B} \rightarrow$ Top $_{0}$, since all groups are pointed at the identity 1 and morphisms preserve identities. By Freyd s theorem $S_{0}$ has a left adjoint $G:$ Top $_{0} \rightarrow \mathfrak{B}$. If $\eta_{0}:\left(X, x_{0}\right) \rightarrow\left(S_{0} G\left(X, x_{0}\right), 1\right)$ is the front adjunction, then for any continuous map $\gamma$ of $X$ into $\mathrm{S}_{0} H$, where $H \in \mathfrak{B}$ and $\gamma\left(x_{0}\right)$ is the identity in $H$, there exists a unique continuous homomorphism $\Gamma$ of $G\left(X, x_{0}\right)$ into $H$ such that $S(\Gamma) \eta_{0}=\gamma$.Using arguments similar to those used for $F(X, \mathfrak{B})$ in [7], we can show that $G\left(X, x_{0}\right)$ is the free group on the set $\left\{x: x \in \eta_{0}(X)-x_{0}\right\}$ of the underlying variety of groups. So $\eta_{0}(X)$ generates $G\left(X, x_{0}\right)$ algebraically. Again we see that $\eta_{0}$ maps $X$ homeomorphically into $\eta_{0}(X)$ if and only if there exists a member $H$ of $\mathfrak{B}$ having a subspace homeomorphic to $X$. [This statement is stronger than we might expect but this is because we are dealing with categories of topological groups. So if $\theta: X \rightarrow H$ is such that $\theta: X \rightarrow \theta(X)$ is a homeomorphism, then by defining $\theta_{0}: X \rightarrow H$ by $\theta_{0}(x)=\theta(x) \theta\left(x_{0}\right)^{-1}$ for each $x \in X$, we see that $\theta_{0}: X \rightarrow \theta_{0}(X)$ is a homeomorphism and $\theta_{0}\left(x_{0}\right)$ is the identity element of $H$.

THEOREM 2. If $\mathfrak{B}$ is a variety and $X$ is a topological space, then $G(X, \mathfrak{B})$ exists if and only if some member of $\mathfrak{B}$ has a subspace homeomorphic to $X$. Further, if $G(X, \mathfrak{B})$ exists then it is unique, up to isomorphism. (In particular, it is independent of the choice of base point.)

Proof. Our above discussion leaves only the last sentence to be verified. We show that if $x_{1}$ and $x_{2}$ are in $X$, the there is an isomorphism $\tau: G\left(X, x_{1}\right)$ $\rightarrow G\left(X, x_{2}\right)$. (We continue the above notation, with $\eta_{i}:\left(X, x_{i}\right) \rightarrow S_{0} G\left(X, x_{i}\right)$, $i=1,2$.)

Define a continuous map $\gamma_{1}: X \rightarrow S_{0} G\left(X, x_{2}\right)$ by $\gamma_{1}(x)=\eta_{2}(x) \eta_{2}\left(x_{1}\right)^{-1}$, for 
all $x \in X$. Then $\gamma_{1}\left(x_{1}\right)$ is the identity element of $G\left(X, x_{2}\right)$. Therefore, there exists a unique continuous homomorphism $\tau: G\left(X, x_{1}\right) \rightarrow G\left(X, x_{2}\right)$ such that $S_{0}(\tau) \eta_{1}=\gamma_{1}$. Similarly we can define a continuous map $\gamma_{2}: X \rightarrow S_{0} G\left(X, x_{1}\right)$ by $\gamma_{2}(x)=$ $\eta_{1}(x) \eta_{1}\left(x_{2}\right)^{-1}$ for all $x \in X$, and there exists a unique continuous homomorphism $\tau^{\prime}: G\left(X, x_{2}\right) \rightarrow G\left(X, x_{1}\right)$ such that $S_{0}\left(\tau^{\prime}\right) \eta_{2}=\gamma_{2}$.

It is easily verified that for each $x \in X, \tau^{\prime} \tau\left(\eta_{1}(x)\right)=\eta_{1}(x)$. Since $\eta_{1}(x)$ generates $G\left(X, x_{1}\right)$ algebraically, $\tau^{\prime} \tau$ acts identically on $G\left(X, x_{1}\right)$. Similarly $\tau \tau^{\prime}$ acts identically on $G\left(X, x_{2}\right)$. Thus $\tau$ is an isomorphism of $G\left(X, x_{1}\right)$ onto $G\left(X, x_{2}\right)$ and the proof is complete.

Now we note that the forgetful functor $T:$ Top $_{0} \rightarrow$ Top has a left adjoint, namely the functor $P$ with $P X=\left(X \cup\{*\},{ }^{*}\right)$, the space obtained by adjoining an isolated base point. Since $T S_{0}: \mathfrak{B} \rightarrow$ Top is just the forgetful functor $S$, then $F$ is naturally isomorphic to GP. Thus we have:

THEOREM 3. If $\mathfrak{B}$ is a variety, then every Markov free topological group of $\mathfrak{B}$ is a Graev free topological group of $\mathfrak{D}$. More precisely each $F(X, \mathfrak{B})$ is isomorphic to $G(Y, \mathfrak{B})$, where $Y$ is the disjoint union of $X$ and a single point.

Our next theorem answers the question: When is a Graev free topological group a Markov free topological group?

THEOREM 4. Let $X$ be a topological space and $\mathfrak{B}$ a variety such that $G(X, \mathfrak{B})$ exists.

(i) If $X$ is connected then $G(X, \mathfrak{B})$ is connected. Consequently, if $\mathfrak{B}$ contains any non-indiscrete group then $G(X, \mathfrak{B})$ is not a Markov free topological group of $\mathfrak{B}$.

(ii) If $X$ is disconnected, then there exists a topological space $K$ such that $G(X, \mathfrak{B})$ is isomorphic to $F(K, \mathfrak{B})$.

ProOF. Let $\eta_{0}: X \rightarrow S_{0} G(X, \mathfrak{Y})$, as before.

(i) Since $\eta_{0}(X)$ is connected and contains the identity element 1 of $G(X, \mathfrak{B})$, the component of 1 contains $\eta_{0}(X)$ and hence is the whole group $G(X, \mathfrak{B})$.

(ii) If 1 is an isolated point of $\eta_{0}(X)$, then by the comments preceding Theorem $3, G(X, \mathfrak{B})$ is isomorphic to $F(X-1, \mathfrak{Y})$. That $G(X, \mathfrak{B})$ is not a Markov free topological group of $\mathfrak{B}$, for $\mathfrak{B}$ non-indiscrete, follows from Theorem 6.1 of [9].

Now assume only that $X$ is disconnected. Then $\eta_{0}(X)=X_{1} \cup X_{2}$ where $X_{1}$ and $X_{2}$ are open subsets of $\eta_{0}(X)$. Let $1 \in X_{1}$ and $f$ be any element of $X_{2}$. Put $Y=\{1\} \cup f X_{1} \cup X_{2}$. It is clear that $G(X, \mathfrak{B})$ is $G(Y, \mathfrak{B})$. To complete the proof we only have to show that 1 is an isolated point of $Y$.

Since $G(X, \mathfrak{B})$ is not indiscrete there is an element $a \in G(X, \mathfrak{B})$ such that a $\notin \mathrm{cl}$. $\{1\}$. Then $1 \in A \subset U$, where $A$ is closed in $G(X, \mathfrak{B}), U$ is open in $G(X, \mathfrak{B})$ and $a$ $\notin U$. Define a mapping $\gamma$ of $X$ into $G(X, \mathfrak{B})$ by $\gamma(x)=1$ if $\eta_{0}(x) \in X_{1}$ and $\gamma(x)=a$ if $\eta_{0}(x) \in X_{2}$. Since $\gamma$ is continuous there exists a continuous homomorphism $\Gamma$ of $G(X, \mathfrak{B})$ into itself such that $\Gamma \eta_{0}=\gamma$. Clearly $\Gamma\left(f X_{1}\right)=\Gamma\left(X_{2}\right)=a$ while $\Gamma(1)=1$. 
So $\Gamma^{-1}(A) \cap Y=\Gamma^{-1}(U) \cap Y=1$. Hence 1 is an isolated point of $Y$. Thus the proof is complete.

In [10] the following is proved:

THEOREM 5. Let $\mathfrak{B}$ be a variety and $\left\{G_{i}: i \in I\right\}$ a family of members of $\mathfrak{B}$. Then $\coprod_{\mathfrak{F}}$ exists and is unique, up to isomorphism.

We note that a $\mathfrak{B}$-product is simply a coproduct in the category $\mathfrak{B}$. Looking at coproducts in our other categories we have: if $\left\{X_{i}: i \in I\right\}$ is a family in Top, then the coproduct $\coprod X_{i}$ is the disjoint union of the $X_{i}$. If $\left\{\left(X_{i}, x_{i}\right) ; i \in I\right\}$ is a family in Top $_{0}$, then the coproduct $\coprod\left(X_{i}, x_{i}\right)$ is the space obtained from the coprodduct in Top by identifying all points $x_{i}$ (when considered in the coproduct) to a single point $x_{0}$. Now we use the well known fact that left adjoints preserve colimits - in particular, coproducts. So we have

$$
\begin{gathered}
F\left(\coprod X_{i}\right)=\coprod_{\mathfrak{B}} F\left(X_{i}\right) \\
G\left(\coprod\left(X_{i}, x_{i}\right)\right)=\coprod_{\mathfrak{B}} G\left(X_{i}, x_{i}\right)
\end{gathered}
$$

Then (2) gives:

THEOREM 6. Let $\mathfrak{B}$ be a variety and $\left\{X_{i}: i \in I\right\}$ a family of topological spaces. In each $X_{1}$ distinguish a point $e_{i}$ and let $Y$ be the free union of the $X_{i}$ with all the $e_{i}$ identified. If $G(Y, \mathfrak{B})$ exists, then it is isomorphic to $\coprod_{\mathfrak{B}} G\left(X_{i}, \mathfrak{B}\right)$.

PROOF. By Theorem 2, the existence of $G(Y, V)$ implies the existence of $G\left(X_{i}, \mathfrak{B}\right)$, for each $i \in I$. The result is then an immediate consequence of statement (2) above.

We could state a similar theorem for Markov free topological groups. However, we first prove a lemma which allows us to prove a stronger version.

Definitron. Let $G$ be a topological group and $X$ a subspace of $G$ which generates $G$ algebraically. Then $G$ is said to be a relatively free topological group with free generating space $X$, if every continuous mapping of $X$ into $G$ can be extended to a continuous endomorphism of $G$. (See [7])

Clearly each $F(X, \mathfrak{B})$ is a relatively free topological group with free generating space $\eta(X)$.

LEMMA. Let $G$ be a relatively free topological group with free generating space $X$. If $G$ is not indiscrete, then the identity element 1 is an isolated point of $Y=X \cup\{1\}$.

Proof. Since $G$ is not indiscrete, there is a $g \in G$ such that $g \notin c l .\{1\}$. Then $g \in A \subset U$, where $A$ is a closed subset of $G, U$ is an open subset of $G$ and $1 \notin U$. Define a mapping $\gamma$ of $X$ into $G$ by $\gamma(X)=g$. Since $\gamma$ is continuous, there exists a continuous homomorphism $\Gamma$ of $G$ into itself such that $\Gamma \mid X=\gamma$. Now $\Gamma(X)=g$ 
and $\Gamma(1)=1$. So $\Gamma^{-1}(A) \cap Y=\Gamma^{-1}(U) \cap Y=X$. Hence 1 is an isolated point of $Y$.

Our next theorem says somewhat more than Theorem 3.10 of [10].

THEOREM 7. Let $\mathfrak{B}$ be a non-indiscrete variety and $\left\{X_{i}: i \in I\right\}$ a family of topological spaces. If $F\left(X_{i}, \mathfrak{B}\right)$ exists for each $i \in 1$, then $\coprod_{\mathfrak{B}} F\left(X_{i}, \mathfrak{B}\right)$ is isomorphic to $F(Y, \mathfrak{B})$, where $Y$ is the free union of the $X_{i}$.

Proof.Using the above lemma we see that $Y$ is homeomorphic to a subspace of $\prod_{i \in I} F\left(X_{\imath}, \mathfrak{B}\right)$. Therefore, by Theorem $1, F(Y, \mathfrak{B})$ exists. It is now clear from earlier statement $(1)$ that $F(Y, \mathfrak{B})$ is isomorphic to []$_{\mathfrak{B}} F\left(X_{i}, \mathfrak{B}\right)$.

Now let $X$ and $Y$ be topological spaces with $x \in X$ and $X \llbracket Y Y$ their free union. Then $(X \coprod Y, x)=(X, x) \coprod(Y \cup\{x\}, x)=(X, x) \coprod P Y$. Hence we have

$$
G(X \amalg Y, x)=G(X, x) \amalg G P Y .
$$

Since the functor $G P$ is naturally isomorphic to $F$, we have

$$
G(X \coprod Y, x)=G(X, x) \amalg F Y .
$$

THEOREM 8. Let $\mathfrak{B}$ be a non-indiscrete variety and $X$ and $Y$ topological spaces such that $F(X, \mathfrak{B})$ and $F(Y, \mathfrak{B})$ exist. If $Z$ is the free union of $X$ and $Y$, then $G(Z, \mathfrak{B})$ is isomorphic to both $F(X, \mathfrak{V}) \coprod_{\mathfrak{B}} G(Y, \mathfrak{B})$ and $G(X, \mathfrak{B}) \coprod_{\mathfrak{B}} F(Y, \mathfrak{B})$.

Proof. It is shown in Theorem 7 that if $F(X, V)$ and $F(Y, V)$ exist, then so does $F(Z, \mathfrak{B})$. Therefore, by Theorem $2, G(Z, \mathfrak{B})$ exists. The result is then an immediate consequence of the above statement (3).

COROLlaRY 1. Let $\mathfrak{B}$ be a non-indiscrete variety and $X$ a topological space If $F(X, \mathfrak{B})$ exists, then it is isomorphic to $G(X, \mathfrak{B}) \coprod_{\mathfrak{B}} F(Y, \mathfrak{B})$, where $Y$ is a onepoint topological space. (cf. [15].)

REMARK 1. The above corollary is of most interest when $\mathfrak{V}$ contains the discrete group $Z$ of integers, in which case $F(Y, \mathfrak{B})$ is isomorphic to $Z$.

COROLLARY 2. If $\mathfrak{B}$ is a variety and $X$ and $Y$ are topological spaces such that $G(X, \mathfrak{B})$ and $G(Y, \mathfrak{B})$ are isomorphic then $F(X, \mathfrak{B})$ and $F(Y, \mathfrak{B})$ are isomorphic.

REMARK 2. As an application of our work we answer a question of Nummela [13].

Let $X$ be a compact group, $F G(X)$ the Graev free topological group on $X$ (in the variety of all topological groups) and $\sigma: F G(X) \rightarrow X$ the canonical quotient morphism. Nummela shows that if $H$ is the kernel of $\sigma$, then $H$ is a Graev free topological group. (Consequently, every compact group has "projective dimension"' one.)

He asks if the above proposition is true with $F G(X)$ replaced by $F M(X)$, the 
Markov free topological group on $X$, and "Graev" replaced by "Markov". The answer is in the affirmative.

We note that $F M(X) \cong F G(X) \backslash \backslash Z$ and if $\sigma^{\prime}$ is the canonical quotient morphism from $F M(X)$ onto $X$, then the kernel of $\sigma^{\prime}$ is $H \coprod Z$ (where $H$ is as above) and consequently is a Markov free topological group, since $H$ is a Graev free topological group.

REMARK 3. Projective topological groups have been studied in [8], [10], [3], [4], [5], [13], [14] and [15]. For our purposes here, we say the topological group $P \in \mathfrak{B}$ is projective in $\mathfrak{B}$ if $P$ is a retract of $F(X, \mathfrak{B})$, for some topological space $X$. We point out that Corollary 1 implies that for any non-indiscrete variety $\mathfrak{B}$, $G(X, \mathfrak{B})$ is a retract of $F(X, \mathfrak{B})$. Thus we see that for any variety $\mathfrak{B}, G(X, \mathfrak{B})$ is projective in $\mathfrak{B}$.

Definition. A variety $\mathfrak{B}$ is said to be a $\beta$-variety if for each Tychonoff space $X, F(X, \mathfrak{B})$ exists and is Hausdorff.

For comments on $\beta$-varieties see [8] and [12].

THEOREM 9. A variety $\mathfrak{B}$ is a $\beta$-variety if and only if $G(X, \mathfrak{B})$ exists and is Hausdorff for each Tychonoff space $X$.

Proof. Let $\mathfrak{B}$ be a $\beta$-variety and $X$ a Tychonoff space. Then $F(X, \mathfrak{B})$ exists and is Haudorff. Corollary 1 implies that $G(X, \mathfrak{B})$ exists and is isomorphic to a sub-group of $F(X, \mathfrak{B})$. Therefore $G(X, \mathfrak{Y})$ is Hausdorff.

Conversely let $\mathfrak{B}$ be a variety such that $G(X, \mathfrak{B})$ exists and is Hausdorff for each Tychonoff space $X$. Let $Y$ be the disjoint union of $X$ and $\{a\}$. Then $Y$ is a Tychonoff space. Consequently $G(Y, \mathfrak{B})$ exists and is Hausdorff. However, by Theorem 3, $G(Y, \mathfrak{B})$ is isomorphic to $F(X, \mathfrak{Z})$; that is, $F(X, \mathfrak{B})$ exists and is Hausdorff.

\section{Acknowledgements}

The author thanks R.L. Cooley for supplying a copy of his Ph.D. dissertation which helped to motivate this study.

Particular thanks go to G.M. Kelly and Saunders Mac Lane for first pointing out how the above material related to category theory. Thanks also to the referee whose very detailed suggestions were invaluable.

\section{References}

[1] Peter Freyd, Abelian Categories (Harper and Row, New York, 1964).

[2] M. I. Graev, 'Free topological groups', Izv. Akad. Nauk SSSR Ser. Mat. 12 (1948), 279-324, (Russian). English transl., Amer. Math. Soc. Transl. no. 35 (1951). Reprint Amer. Math. Soc. Transl. (1) 8(1962), 305-364.

[3] C. E. Hall, 'Projective topological groups', Proc. Amer. Math. Soc. 18 (1967), 425-431.

[4] C. E. Hall, 'F-projective groups', Proc. Amer. Math. Soc. 26 (1970), 193-195. 
[5] Karl Heinrich Hofmann, 'Zerfällung topologischer Gruppen', Math. Z. 84 (1964), 16-37.

[6] A. A. Markov, 'On free topolog:cal groups', C. R. (Doklady) Acad. Sci. URSS, (N.S.) 31 (1941), 299-301. Bull. Acad. Sci. URSS Sér. Math. [lzv. Akad. Nauk. SSSR] 9 (1945), 3-64. (Russian-English summary) English Transl. Amer. Math. Soc. Transl. no. 30 (1950), 11-88; reprint Amer. Math. Soc. Transl. (1) 8(1962), 195-272.

[7] Sidney A. Morris, 'Varieties of topological groups', Bull. Austral. Math. Soc. 1(1969), 145-160.

[8] Sidney A. Morris, 'Varieties of topological groups II', Bull. Austral. Math. Soc. 2 (1970), 1-13.

[9] Sidney A. Morris, 'Varieties of topological groups III', Bull. Austral. Math. Soc. 2 (1970), 165-178.

[10] Sidney A. Morris, 'Free products of topological groups', Bull. Austral. Math. Soc. 4 (1971), 17-29.

[11] Sidney A. Morris, 'Free compact abelian groups', Mat. Casopis. Sloven. Akad. Vied. 22 (1972).

[12] Sidney A. Morris, 'Locally compact groups and $\beta$-varieties of topological groups,' Fund. Math. (to appear).

[13] Hanna Neumann, Varieties of Groups (Ergebnisse der Mathematik und ihrer Grenzgebiete, Band 37, Springer-Verlag, Berlin, Heidelberg, New York, 1967.)

[14] Eric C. Nummela, 'The projective dimension of a compact abelian groups', (to appear).

[15] Eric C. Nummela, 'Homological algebra of topological modules', (to appear).

[16] F. R. Ward, 'On free and projective topological groups', Notices Amer. Math. Soc. 17 (1970), 135.

University of New South Wales

Kensington 2033, Australia 